\title{
PERFIL DOS USUÁRIOS DE UM CENTRO DE ATENÇÃO PSICOSSOCIAL INFANTOJUVENIL DA GRANDE SÃO PAULO, BRASIL
}

\section{PROFILE OF PATIENTS FROM A PSYCHOSOCIAL CARE CENTRE FOR CHILDREN AND ADOLESCENTS IN SÃO PAULO CITY, BRAZIL}

\author{
Patrícia Santos de Souza Delfini ${ }^{1}$ \\ Caroline Dombi-Barbosa ${ }^{2}$ \\ Felipe Lessa da Fonseca ${ }^{3}$ \\ Carlos Mendes Tavares ${ }^{4}$ \\ Alberto Olavo Advicula Reis ${ }^{5}$
}

Delfini PSS et al. Perfil dos usuários de um centro de atenção Psicossocial infantojuvenil da grande São Paulo, Brasil. Rev Bras Crescimento Desenvolv Hum. 2009; 19(2):226-236.

\section{Resumo:}

Introdução: os Centros de Atenção Psicossocial Infantojuvenil (CAPSi) constituem ponta de lança das ações da Reforma Psiquiátrica Brasileira e têm por finalidade o atendimento de crianças e adolescentes com transtornos psíquicos graves. O objetivo é caracterizar o perfil dos usuários de um CAPSi, considerando sexo, idade, hipótese diagnóstica, origem do encaminhamento, inserção escolar e motivo de consulta. Método: por meio de um protocolo, foram coletados dados da totalidade de prontuários ativos de uma unidade da Grande São Paulo - cento e três - no mês de janeiro de 2008. Resultados: a maioria dos usuários atendidos está na faixa etária de cinco a quinze anos (68,9\%) e é do sexo masculino (61,2\%). O grupo de transtornos de comportamento e transtornos emocionais corresponde a $21,4 \%$, seguido pelos transtornos do desenvolvimento global $(16,2 \%)$ e retardo mental (10,5\%). A maioria dos usuários foi encaminhada pelo Conselho Tutelar (22,3\%) e tiveram como principal motivo da consulta queixas neuromotoras (17,5\%), escolares $(15,5 \%)$ e sociocomportamentais (14,6\%). Conclusões: o número elevado de crianças com problemas neuromotores pode indicar características específicas da instituição estudada que absorveu pacientes e profissionais de um antigo serviço de reabilitação. O grande número de questões relevantes não encontradas apontam para a falta de padronização dos prontuários.

Palavras-chave: saúde mental; unidade pública de saúde; infância; adolescência; perfil dos usuários.

Trabalho resultante do processo FAP/ESP nº 2006/06902-2-2, realizado pelo Laboratório de Saúde Mental Coletiva (LASAMEC), da Faculdade de saúde Pública da USP.

1 Psicóloga. Mestranda do Depto. de Saúde Materno-Infantil da Faculdade de Saúde Pública da USP. Pesquisadora do LASAMEC. Bolsista CNPq - Brasil. E-mail: patriciadelfini@usp.br

2 Psicóloga. Mestre em Saúde Pública. Depto. de Saúde Materno-Infantil da Faculdade de Saúde Pública da USP. Pesquisadora do LASAMEC. E-mail: caroldombi@usp.br

3 Psicólogo. Doutor pela Pontifícia Universidade Católica/SP. Pesquisador do LASAMEC. E-mail: flessaf@uol.com.br

4 Estatístico, doutor em Saúde Pública pela Faculdade de Saúde Pública da USP. E-mail: carlostavares@usp.br

5 Professor Doutor, orientador do Depto. de Saúde Materno-Infantil da Faculdade de Saúde Pública da USP. Coordenador do LASAMEC. E-mail: albereis@usp.br 


\begin{abstract}
:
Psychosocial Care Centres for children and adolescents (CAPSi) are the main services concerning Brazilian Psychiatric Reform and aim to the assistance of children and adolescents suffering from persistent psychic diseases. The objective of the paper is to describe the profile of patients of a CAPSi, considering sex, age, diagnosis, kind of referral, school insertion and reason for searching for the service. Method: data have been collected from all active patients files from a unit from São Paulo state, Brazil - one hundred and three - through a protocol. This paper shows partial data from a project entitled Epidemiologic and Social demographic characteristics of patients of the CAPSi from São Paulo state. Results: most patients assisted in this service are between five and fifteen years old (68,9\%) and are male (61,2\%). Behavioural and emotional disorders afected $21,4 \%$ of all patients, followed by disorders of psychological development $(16,2 \%)$ and mental retardation (10,5\%). Patients were referred to the unit mostly by State Child Protection Agency (22,3\%) and had motor neuronal problems (17,5\%), school difficulties $(15,5 \%)$ and social behavior matters $(14,6 \%)$ as the main reasons for searching for assistance.

Conclusions: the high number of motor neuronal problems may indicate specific characteristics of the unit, which has taken patients and professionals from an older outpatient rehabilitations service. The great number of relevant characteristics were not found in patients files may show lack of standard in registration.
\end{abstract}

Key words: mental health; Psychosocial Care Centres; childhood; adolescence; patients' profile.

\section{INTRODUÇÃO}

Os Centros de Atenção Psicossocial Infantojuvenis (CAPSi) visam a atender crianças e adolescentes portadores de sofrimento psíquico grave e persistente, de modo que suas ações possam ajudá-los em sua inserção social. A Organização Mundial de Saúde ${ }^{1}$ estima que os problemas relacionados ao sofrimento psíquico representam cinco das dez principais causas de incapacidade no mundo. Cerca de 400 milhões de habitantes do planeta sofrem de transtornos mentais ou neurológicos ou ainda de problemas psicossociais associados, por exemplo, ao abuso de álcool ou drogas.

Os transtornos mentais na infância e adolescência têm forte impacto social e familiar e podem estar associados a problemas psiquiátricos e sociais ao longo da vida. ${ }^{2}$ A prevalência desses transtornos estimada para a população infantojuvenil vai de $10 \%$ a $15 \%^{3,4}$, Hoffmann ${ }^{5}$ amplia a faixa de prevalência até os $20 \%$ e considera os mais comuns como sendo os de comportamento, atenção, emocionais e hiperatividade.

Um estudo epidemiológico realizado na década de 80, na Bahia, apontou a prevalência de transtornos infantis em 23,2\% das crianças estudadas, sendo que $10 \%$ eram casos graves e moderados e $13,2 \%$, transtornos mentais leves ou duvidosos que dispensavam atendimento psicológico. ${ }^{6}$

Pesquisa semelhante realizada em Embu, município da Grande São Paulo, constatou que $24,6 \%$ das crianças e adolescentes investigados apresentaram algum tipo de transtorno mental, sendo que $7,3 \%$ necessitavam de atenção especializada devido a transtornos mais graves. ${ }^{7}$ 
Vitolo et $\mathrm{al}^{8}$, em estudo na cidade de Taubaté, interior de São Paulo, concluíram que $35,2 \%$ da população inscrita nas três primeiras séries do ensino fundamental foram consideradas como positivas para problemas de saúde mental, sendo os sintomas mais frequentes a ansiedade e depressão (47,8\%), problemas de conduta (32,6\%) e hiperatividade e déficit de atenção $(31,1 \%)$.

Lauridsen Ribeiro ${ }^{9}$ desenvolveu um estudo com 141 crianças e adolescentes entre um e dezenove anos de idade para avaliar queixas referidas a "problemas de nervos" e detectou a prevalência de 4,7\%. Desses, 74\% não buscaram qualquer tipo de ajuda, e dos casos que procuraram, 55,6\% consultaram profissionais médicos em centros de saúde ou hospital geral e, em $12,9 \%$ dos casos, o profissional procurado foi o psicólogo.

Algumas dificuldades para identificação de transtornos mentais na população infantojuvenil ${ }^{10}$ são: a indefinição de normal e patológico para determinada idade e contexto sociocultural; as variações qualitativas e quantitativas de cada sintoma; o fator do desenvolvimento, a implicação em avaliar algo que $a$ priori está em transformação. Outras variáveis que podem interferir na avaliação de sintomas são a percepção do informante e suas características (pais, professores e vizinhos terão percepções distintas acerca de uma mesma problemática); os métodos e tipos de instrumentos para avaliação usados por diferentes profissionais que nem sempre são padronizados, o que gera diferentes classificações.

As conseqüências dos transtornos mentais e sua importância para população estiveram, por muito tempo, ausentes das agendas de políticas públicas em todo o mundo. A saúde mental infantojuvenil, em especial, foi durante muito tempo tratada numa conjuntura estreitamente filantrópica e assistencialista. A revista "The Lancet" (2007) ${ }^{11}$ lançou um número especial que destacou o fato de não ha- ver saúde sem saúde mental. No Brasil, a resposta dada à lacuna histórica de atenção pública à saúde mental infantojuvenil foi representada pela criação dos Centros de Atenção Psicossociais (CAPS). A partir da portaria 336/ $02^{12}$ foram constituídos os Centros de Atenção Psicossociais Infantojuvenis (CAPSi) que passaram a ser um importante dispositivo social para o atendimento de crianças e adolescentes que apresentam grave e persistente transtorno mental.

Além disso, é preconizado que o atendimento ao sujeito que apresenta sofrimento psíquico possa encontrar respaldo numa rede articulada de serviços de saúde. Nesse sentido, o CAPS é um dos equipamentos que, inserido no território e articulado a todos os recursos disponíveis, deve buscar contribuir para a ampliação da autonomia e inserção social da população.

No entanto, na prática, esses serviços deparam-se com situações diversificadas que não raro se distanciam de suas diretrizes. Consequentemente, a caracterização do ponto de vista sócio-demográfico e epidemiológico é de interesse e importância na medida em que permite conhecer a real situação à qual eles efetivamente respondem.

O levantamento de características da população atendida nos serviços da rede de saúde mental representa uma contribuição objetiva e importante para uma reflexão acerca da distância existente entre as diretrizes que orientam esses equipamentos e a realidade cotidiana com a qual se deparam. Desta forma, a descrição e análise do perfil dos usuários desses serviços permitem que se constituam indicadores da adequação das diretrizes dos CAPSis à realidade local desses equipamentos.

O objetivo do estudo é caracterizar o perfil dos usuários atendidos em um Centro de Atenção Psicossocial Infantojuvenil da Grande São Paulo. 


\section{MÉTODO}

Trata-se de estudo piloto, descritivo, transversal, realizado com dados obtidos por meio dos prontuários dos usuários de um Centro de Atenção Psicossocial Infantojuvenil (CAPSi) da Grande São Paulo. Os dados foram coletados no mês de janeiro de 2008.

A escolha da população envolveu o universo dos prontuários que se encontravam ativos no momento da coleta, totalizando 103 prontuários. Foram considerados ativos os prontuários dos usuários que freqüentassem o serviço naquele mês e que estivessem matriculados no CAPSi há, no mínimo, três meses.

Para coleta de dados foi construído um protocolo de pesquisa composto pelos seguintes itens: sexo, idade, hipótese diagnóstica, origem do encaminhamento, inserção escolar e motivo da consulta.

A pesquisa cumpre as exigências éticas da resolução 196 (1996) e foi aprovada pelo Comitê de Ética em Pesquisa da Faculdade de Saúde Pública (protocolo 1616 de 20 de abril de 2007) e pelo Comitê de Ética do município onde foi realizada.

\section{RESULTADOS E DISCUSSÃO}

Os usuários atendidos no Centro de Atenção Psicossocial Infantojuvenil (CAPSi) estudado são em sua maioria do sexo masculino (61,2\%). Essa maior prevalência coincide com a informação presente na pesquisa de Hoffmann $^{13}$, na qual a maior parte da população atendida em sete CAPSi de diferentes regiões do país são do sexo masculino bem como na de Santos et al ${ }^{14}$, que indica serem crianças e adolescentes do sexo masculino a maioria atendida em ambulatórios de saúde mental da Bahia.

Constatou-se que a maioria dos usuários tem entre cinco e quinze anos (68,9\%), sendo a média de idade de 9,43 anos ( $D P=4,6$; variação de 1 a 19). Essa média é semelhante a encontrada em outras unidades CAPSi do país $^{13}$. Entre os meninos, predomina a faixa de 10 a 15 anos (39,7\%), enquanto as meninas têm entre 5 e 10 anos (37,5\%), conforme indicado na tabela 1.

No que se refere ao perfil diagnóstico, observa-se que grande parte dos prontuários não possui o registro da hipótese diagnóstica (24,8\%). Levando em conta a Classificação Internacional das Doenças, CID-10 $0^{15}$, 21\% dos usuários estão no grupo de transtornos de comportamento e transtornos emocionais, $16,2 \%$ no grupo de transtornos do desenvolvimento psicológico e 10,5\% apresentam retardo mental. A pesquisa de Hoffmann ${ }^{13}$ também encontra os transtornos de comportamento e emocionais,

Tabela 1: Distribuição do número (n) e proporção (\%) dos usuários do CAPSi segundo faixa etária e sexo, Grande São Paulo, 2008.

\begin{tabular}{ccccc}
\hline Faixa Etária & & Feminino & \multicolumn{2}{c}{ Masculino } \\
\hline & $\mathbf{n}$ & $\mathbf{\%}$ & $\mathbf{n}$ & $\%$ \\
\hline 1 I-5 & 8 & 20,0 & 9 & 14,3 \\
$5 \mathrm{I}-10$ & 15 & 37,5 & 22 & 34,9 \\
$10 \mathrm{I}-15$ & 9 & 22,5 & 25 & 39,7 \\
$15 \mathrm{I}-20$ & 8 & 20,0 & 7 & 11,1 \\
Total & 40 & 100,0 & 63 & 100,0 \\
\hline
\end{tabular}

- Fonte: REIS AOA. Pesquisa Caracterização Epidemiológica e Sócio-demográfica dos Centros de Atenção Psicossociais Infantojuvenis do Estado de São Paulo, processo FAPESP 06/06902-2. 
bem como o transtorno do desenvolvimento global com grande freqüência.

Ao associar a hipótese diagnóstica com o sexo, notam-se algumas diferenças. No caso dos meninos, o diagnóstico mais frequente é de transtorno de comportamento e emocionais (25,4\%), embora esse também tenha destaque no sexo feminino (15\%). Já para as meninas, o retardo mental aparece como hipótese diagnóstica mais frequente, presente em $20 \%$ dos casos, enquanto para os meninos essa hipótese corresponda a apenas $4,8 \%$. Muito provavelmente a perspectiva de gênero possa contribuir para a elucidação dessas variações. Os transtornos do desenvolvimento psicológico e as síndromes paralíticas tem destaque para ambos os sexos, como se observa na tabela 2. Não fo-

Tabela 2: Distribuição do número (n) e proporção (\%) dos usuários do CAPSi segundo hipótese diagnóstica e sexo, Grande São Paulo, 2008.

\begin{tabular}{|c|c|c|c|c|c|}
\hline \multirow[t]{2}{*}{$\begin{array}{l}\text { Hipótese } \\
\text { diagnóstica }\end{array}$} & \multirow[t]{2}{*}{ Descrição } & \multicolumn{2}{|c|}{ feminino } & \multicolumn{2}{|c|}{ masculino } \\
\hline & & $\mathrm{n}$ & $\%$ & $\mathrm{n}$ & $\%$ \\
\hline F30-F39 & transtornos de humor (afetivos) & 1 & 2 & - & - \\
\hline F40-F48 & $\begin{array}{l}\text { transtornos neuróticos } \\
\text { transtornos relacionados com estresse e } \\
\text { transtornos somatoformes }\end{array}$ & 2 & 5,0 & - & - \\
\hline F50-F59 & $\begin{array}{l}\text { síndromes comportamentais associadas } \\
\text { com distúrbios fisiológicos e fatores físicos }\end{array}$ & 1 & 2,5 & - & - \\
\hline F70-F79 & retardo mental & 8 & 20,0 & 3 & 4,8 \\
\hline F80-F89 & transtornos do desenvolvimento psicológico & 3 & 7,5 & 14 & 22,2 \\
\hline F90-F98 & $\begin{array}{l}\text { transtornos do comportamento e transtornos } \\
\text { emocionais que aparecem habitualmente na } \\
\text { infância ou na adolescência }\end{array}$ & 6 & 15,0 & 16 & 25,4 \\
\hline G40- G47 & transtornos episódicos e paroxísticos & 1 & 2,5 & - & 0,0 \\
\hline G80-G83 & paralisia cerebral e outras síndromes paralíticas & 3 & 7,5 & 7 & 11,1 \\
\hline G90-G99 & outros transtornos do sistema nervoso & - & - & 1 & 1,6 \\
\hline Q02 & microcefalia & 2 & 5,0 & - & - \\
\hline Q80 & ictiose congênita & - & - & 2 & 3,2 \\
\hline Q90 & síndrome de Down & 2 & 5,0 & 5 & 7,9 \\
\hline Não consta & & 11 & 27,5 & 15 & 23,8 \\
\hline Total & & 40 & 100,0 & 63 & 100,0 \\
\hline
\end{tabular}

- Fonte: REIS AOA. Pesquisa Caracterização Epidemiológica e Sócio-demográfica dos Centros de Atenção Psicossociais Infantojuvenis do Estado de São Paulo, processo FAPESP 06/06902-2. 
ram encontradas diferenças ao se associar a hipótese diagnóstica com a faixa etária.

No que se refere à inserção escolar, foram consideradas apenas as crianças com seis anos ou mais que é a idade limite obrigatória para o início da vida escolar, de acordo com indicações do Conselho Nacional de Educação do Ministério da Educação. ${ }^{16}$ Assim, foram excluídas da análise 22 crianças que tinham cinco anos ou menos no momento da coleta dos dados. Além dessas, outros 10 prontuários foram excluídos por não possuírem a informação em tela. Dentre as analisadas ( $\mathrm{n}=71)$, 83,1\% frequentam escola regular, 7\% estão em escola especial e 9,9\% não frequentam escola (tabela 3). Essas taxas são similares às encontradas para a população geral, de acordo com a Pesquisa Nacional por Amostra de Domicílio ${ }^{17}$, desenvolvida pelo Instituto Brasileiro de Geografia e Estatística, o que indica que as crian-

Tabela 3: Distribuição do número (n) e proporção (\%) dos usuários* do CAPSi segundo inserção escolar, Grande São Paulo, 2008.

\begin{tabular}{ccc}
\hline Inserção escolar & $\mathbf{n}$ & $\mathbf{\%}$ \\
\hline Escola regular & 59 & 83,1 \\
Escola especial & 5 & 7,0 \\
não freqüenta & 7 & 9,9 \\
\hline Total & 71 & 100,0 \\
\hline
\end{tabular}

* Excluídos aqueles com idade de cinco anos ou menos e os prontuários que não constavam a informação.

- Fonte: REIS AOA. Pesquisa Caracterização Epidemiológica e Sócio-demográfica dos Centros de Atenção Psicossociais Infantojuvenis do Estado de São Paulo, processo FAPESP 06/06902-2.

ças atendidas neste CAPSi estão inseridas nas escolas, independente de seus diagnósticos. Não foram constatadas diferenças ao se associar sexo e inserção escolar.
Em relação à origem do encaminhamento, a maioria dos usuários veio por meio do conselho tutelar (22,3\%), 10,7\% foram encaminhados por outros profissionais de saúde,

Tabela 4: Distribuição do número (n) e proporção (\%) dos usuários do CAPSi segundo origem do encaminhamento, Grande São Paulo, 2008.

\begin{tabular}{ccc}
\hline Origem do encaminhamento & $\mathbf{n}$ & $\mathbf{\%}$ \\
\hline AACD/APAE & 3 & 2,9 \\
abrigo & 6 & 5,8 \\
ambulatório & 10 & 9,7 \\
centro especializado de criança e adolescente & 4 & 3,9 \\
conselho tutelar & 23 & 22,3 \\
escola & 7 & 6,8 \\
espontânea & 4 & 3,9 \\
hospital & 2 & 1,9 \\
outros profissionais de saúde & 11 & 10,7 \\
serviço de saúde mental & 5 & 4,9 \\
UBS & 7 & 6,8 \\
não consta & 21 & 20,4 \\
\hline Total & 103 & 100,0
\end{tabular}

- Fonte: REIS AOA. Pesquisa Caracterização Epidemiológica e Sócio-demográfica dos Centros de Atenção Psicossociais Infantojuvenis do Estado de São Paulo, processo FAPESP 06/06902-2. 
como ortopedista, pediatra e neurologista, sem especificação se pertencentes ao serviço público, particular ou conveniado. Embora o modelo de rede de saúde pública preveja que a porta de entrada do sistema de saúde seja a Unidade Básica de Saúde, apenas 6,8\% dos usuários do serviço estudado são provenientes dessas unidades. Vale destacar que $20,4 \%$ dos prontuários não possuem essa informação (tabela 4).

No que se refere ao principal motivo da consulta, verificou-se que $17,5 \%$ apresentaram queixas relacionadas a lesão ou transtorno neuromotor, $15,5 \%$ queixa escolar e $14,6 \%$ agressividade e problemas sociocomportamentais.

O grande número de crianças com queixas neuromotoras ocorre, provavelmente, devido à herança de pacientes e profissionais que essa unidade recebeu do fechamento de um antigo serviço de reabilitação da cidade. As queixas escolares e sociocomportamentais encontradas com grande frequência nos prontuários coincide com o verificado em outras pesquisas e pode indicar não só a dificuldade das famílias e escolas em lidar com crianças e adolescentes que apresentam comportamentos que fogem de seu controle, como também a falta de articulação entre as escolas e o setor saúde.

Constataram-se diferenças importantes entre o sexo e o principal motivo de consulta. No caso dos meninos $20,6 \%$ apresentaram queixa escolar como principal motivo, seguido de lesões ou transtornos neuromotores $(17,5 \%)$ e agressividade ou problema sociocomporta-mental (17,5\%). Já crianças e adolescentes do sexo feminino apresentam como principal motivo de consulta lesões ou transtornos neuromotores (17,5\%), seguido por ansiedade e isolamento, comprometimento de linguagem e fala e vítimas de negligência, abandono e maus-tratos, cada grupo com $12,5 \%$ (tabela 5 ).

Na faixa etária de 0 a 5 anos, tanto meninos quanto meninas exibem como principal motivo de consulta lesões ou transtornos neuromotores. Tal fato parece estar ligado à história da própria instituição que funcionava como um Centro de Reabilitação Infantil, que foi reestruturado e modificado para atender as exigências da Portaria $336 / 2002^{12}$, quando passou a funcionar como CAPSi e absorveu a clientela inicial.

Entre 11 e 15 anos, ambos os sexos apresentam agressividade e problemas sociocomportamentais e, de 16 a 20 anos, o motivo inicial estava relacionado à queixa escolar. Resultados similares foram encontrados no estudo realizado por Schoen-Ferreira et al ${ }^{18}$ num serviço especializado para adolescentes. Os autores concluíram que as principais queixas entre 10 e 19 anos estão primeiramente as queixas escolares, seguidas pelas queixas de desobediência em casa e na escola.

As categorias que se referem ao motivo da consulta foram obtidas por meio da leitura das respostas abertas. Assim, estabeleceu-se um conjunto de 11 categorias referentes aos motivos de consulta. Na categoria lesão/transtorno neuromotor estão incluídos casos nos quais há procura por atendimento especializado, tais como fisioterapia e fonoaudiologia, além de casos com queixas explícitas de atraso no desenvolvimento motor, dificuldade para andar, paraplegia e outras queixas ligadas a questão neuromotora com sinais evidentes desde a tenra infância.

Como queixa escolar foram consideradas as situações de dificuldade ou atraso de aprendizagem, falta de rendimento e de produtividade na escola bem como inadequação comportamental na escola, que envolvem comportamentos de rebeldia, agressividade, má conduta, agitação, hiperatividade, apatia e falta de participação que aparecem predominantemente no contexto escolar. As queixas relacionadas ao desempenho escolar também foram notadas por Linhares et al. ${ }^{19}$

Na pesquisa de Cabral e Sawaia ${ }^{20}$ as conclusões apontam que os psicólogos de um serviço de saúde de Ribeirão Preto, Estado de São Paulo, embora entendam a escola como 
participante, a consideram também como produtora das dificuldades escolares. Apesar disso, eles continuam atendendo essas queixas de forma individualizada e distante do contexto escolar.

A categoria agressividade e problemas sociocomportamentais engloba queixas de dificuldades de relacionamento interpessoal, rebeldia, envolvimento em brigas, situações de fuga, furto, mentira, falta de limites e auto ou hetero-agressividade em diversos contextos, podendo incluir a escola, mas não se restringindo a ela.

Vale destacar que agressividade ou problemas sociocomportamentais nem sempre configuram quadros patológicos, podendo se caracterizar como passageiros ou reativos de modo que é importante não patologizar a agressividade. Nesse sentido, levanta-se a hipótese de que tanto as famílias, quanto as escolas e os profissionais do CAPSi podem reforçar precon- ceitos relacionados à saúde mental e periculosidade ou violência. Como categoria de ansiedade e isolamento foram consideradas respostas como ansiedade propriamente dita, dificuldade ou falta de interação no contato social e familiar, isolamento e angústia.

Na categoria comprometimento de linguagem e fala, presente em 5,8\% do total dos prontuários, encontrou-se casos de procura por atendimento de fonoaudiologia, queixas de ecolalia e de ausência ou fala sem função comunicativa.

A fala é um elemento importante na vida humana porque condutora de sociabilidade, diferenças relacionadas a essa função são facilmente reconhecidas pelos pais e pelo entorno, por isso não raro transtornos de fala se traduzem rapidamente em procura por atendimento de saúde mental.

Alguns distúrbios de linguagem ficam relacionados à função do pensamento e da

Tabela 5: Distribuição do número (n) e proporção (\%) dos usuários do CAPSi segundo motivo da consulta e sexo, Grande São Paulo, 2008.

\begin{tabular}{ccccc}
\hline Motivo da consulta & \multicolumn{2}{c}{ Feminino } & \multicolumn{2}{c}{ Masculino } \\
& n & $\mathbf{\%}$ & n & \% \\
\hline abrigamento & 3 & 7,5 & - & - \\
atraso no desenvolvimento neuropsicomotor & 2 & 5,0 & 6 & 9,5 \\
agressividade/problemas sociocomportamentais & 2 & 5,0 & 11 & 17,5 \\
ansiedade/isolamento & 5 & 12,5 & 4 & 6,3 \\
comprometimento de linguagem e fala & 5 & 12,5 & 1 & 1,6 \\
convulsão/epilepsia & 1 & 2,5 & 2 & 3,2 \\
lesões/transtornos neuromotores & 7 & 17,5 & 11 & 17,5 \\
motivo impreciso/não consta & 4 & 10,0 & 7 & 11,1 \\
queixa escolar & 4 & 10,0 & 13 & 20,6 \\
síndrome & 2 & 5,0 & 4 & 6,3 \\
vítima de violência, negligência ou maustratos & 5 & 12,5 & 4 & 6,4 \\
\hline Total & $\mathbf{4 0}$ & $\mathbf{1 0 0 , 0}$ & $\mathbf{6 3}$ & $\mathbf{1 0 0 , 0}$ \\
\hline
\end{tabular}

- Fonte: REIS AOA. Pesquisa Caracterização Epidemiológica e Sócio-demográfica dos Centros de Atenção Psicossociais Infantojuvenis do Estado de São Paulo, processo FAPESP 06/06902-2. 
associação lógica e podem ser confundidos com autismo, retardo mental ou outros quadros psicopatológicos.

Já na categoria violência, abuso e maus tratos inclui casos nos quais a procura pelo serviço se dá por consequência de vivência de negligência familiar, abandono, suspeita ou abuso sexual e violência doméstica. Autores como Assis et $\mathrm{al}^{21}$ apontam que a atenção à questão da violência contra a população infantojuvenil é fragmentada e exige ações vinculadas à promoção de saúde.

As demais categorias encontradas, conforme apresentadas na tabela 5, foram: motivos imprecisos, correspondentes a $21,1 \%$ do total e que agrupa as queixas indefinidas e também casos em que a informação não foi encontrada no prontuário, indicando a má qualidade de seu preenchimento. Atraso no desenvolvimento neuropsicomotor $(7,8 \%)$ inclui os prontuários em que aparecem as expressões neuropsicomotor associados ou não ao atraso na fala, dificuldade psicomotora e desenvolvimento geral lento.

A categoria "síndrome" aglutina os casos em que o motivo do encaminhamento se expressa pelos diagnósticos síndrome de Down e de Angelman. Algumas síndromes implicam atraso no desenvolvimento neuromotor ou linguístico como sintomatologia associada e, por isso, são encaminhadas a diversos serviços de saúde, dentre os quais aqueles voltados especificamente à questão da saúde mental.

A categoria abrigamento foi aplicada aos casos de crianças abrigadas, que eram atendidas no CAPSi sem apresentarem uma queixa explícita de sofrimento psíquico no prontuário.

\section{CONSIDERAÇÕES FINAIS}

Os dados encontrados em relação ao sexo, faixa etária e principais hipóteses diagnósticas vão ao encontro dos achados na literatura, o que indica que o serviço atende o preconizado pela política pública de saúde mental infantojuvenil ${ }^{12,21}$.

O grande número de encaminhamentos via Conselho Tutelar aponta para a necessidade de uma investigação mais aprofundada, uma vez que algumas crianças são encaminhadas ao Centro de Atenção Psicossocial (CAPSi) sem uma queixa que justifique a medida, apenas aparentemente pelo fato de serem ou estarem abrigadas.

O vasto número de queixas relacionadas à vida escolar mostra a necessidade do desenvolvimento de ações conjuntas com a escola para que essas questões sejam tratadas em outra esfera de atenção que não de um serviço especializado como o CAPSi, ou ainda que essa população possa receber atendimento na atenção básica.

A grande incidência de questões neurológicas e motoras apresentadas como queixa principal pode indicar uma peculiaridade do serviço, que funcionava anteriormente como centro de reabilitação infantil e, em função de seu fechamento, o CAPSi absorveu sua clientela.

A importante ausência de dados como hipótese diagnóstica, origem do encaminhamento e inserção escolar revelada pela presente pesquisa demonstra falta de padronização e falhas nos registros dos prontuários.

Algumas limitações do estudo decorrem do fato de ter sido realizado em apenas uma unidade, muito embora isso o torne significativo para o contexto sócio-administrativo em que foi realizada.

Além disso, o presente artigo originou-se de uma ação investigativa de projeto piloto, sendo possível observar falhas no instrumento e em sua aplicação, que serviram para realizar ajustes e aprimorar a continuidade da pesquisa proposta.

Espera-se poder futuramente cotejar esses dados extraídos do projeto piloto com aqueles a serem obtidos no conjunto dos CAPSis do Estado de São Paulo. 


\section{AGRADECIMENTOS}

Agradecemos o apoio financeiro da Fundação de Amparo à Pesquisa do Estado de São Paulo (FAPESP) no projeto 2006/06902-2, intitulado Caracterização Epidemiológica e Sócio-Demográfica dos Centros de Atenção Psicossocial Infantojuvenis do Estado de São Paulo, relacionado a este artigo.

\section{REFERÊNCIAS}

1. Organização Mundial da Saúde. Classificação estatística internacional de doenças e problemas relacionados à saúde, 10a revisão. v. 1. São Paulo: Centro Colaborador da OMS para a Classificação de Doenças em Português; 1995.

2. Halpern R, Figueiras ACM. Influências ambientais na saúde mental da criança.

J. Pediatr. 2004; 80 (2) 104-110.

3. Organização Mundial da Saúde.

Relatório Mundial da saúde 2001: saúde mental: novas concepções, nova esperança. Lisboa: 2002, p. 208.

4. Brasil. Ministério da Saúde. Secretaria de políticas de Saúde. Área de Saúde do Adolescente e do Jovem. Cadernos, juventude, saúde e desenvolvimento, v. 1. Brasília, DF, agosto, 1999, 303 p.

5. Hoffmann MCCL. Perfil demográfico e clínico da clientela atendida nos Centros de Atenção Psicossocial Infantojuvenil (CAPSi) no ano de 2003 [dissertação de mestrado]. Bahia: Instituto de Saúde Coletiva da Universidade Federal da Bahia, 2005.

6. Almeida Filho N, Santana VS, Abnoel 1, Jacobina RR. Relações entre a saúde mental dos pais e a saúde mental de crianças em uma população de SalvadorBahia. Acta psiquiátr. psicol. Am. Lat. 1985; 31(3):211-21.

7. Paula CS, Duarte CS, Bordin IAS. Prevalência de problemas de saúde mental em crianças e adolescentes da região metropolitana de São Paulo: necessidade de tratamento e capacidade

de atendimento. Rev. Bras. Psiquiatr. 2007; 29 (1): 11-17.

8. Vitolo YLC, Fleitlich-Bilyk RG, Bordin IAS. Crenças e atitudes educativas dos pais e problemas de saúde mental em escolares. Rev. Saúde Pública. 2005; (39): 716-724.

9. Lauridsen EP. Atenção à saúde mental infantil: um desafio às práticas de saúde - um olhar epidemiológico [dissertação de mestrado]. São Paulo: Faculdade de Saúde Pública da USP; 1998.

10. Lauridsen-Ribeiro EPP, Tanaka OY. Problemas de saúde mental das crianças: abordagens na Atenção Básica. São Paulo: Annablume, 2005.

11. The Lancet. Series Global mental health, 2007. Disponível em: URL:HTTP:// www.thelancet.com/collections/series/ mentalhealeth. Acesso em 15/4/2008.

12. Ministério da Saúde. Portaria/GM n ${ }^{\circ} 336$ de 19 de fevereiro de 2002. Brasília, 2002.

13. Hoffmann MCCL, Santos DN, Mota ELA. Caracterização dos usuários e dos serviços prestados por Centros de Atenção Psicossocial Infantojuvenil. Cad. Saúde Pública. 2008; 24 (3): 633-642.

14. Santos DN, Carvalho MM, Pinho MA, Nunes APR. Atendimento em psiquiatria da infância e adolescência em serviços públicos de Salvador. Revista Baiana de Saúde Pública. 2005; v.29 n.1, p.35-42.

15. Organização Mundial de Saúde. Classificação de Transtornos Mentais e do Comportamento da CID 10. Porto Alegre: Artes Médicas; 1998. 
16. Ministério da Educação (MEC).

Conselho Nacional da Educação, 2009.

Conselho Site: http://

www.deolhonaeducacao.org.br/

Comunicacao. aspx?action $=5 \& \mathrm{mID}=4108$

acesso em 01/08/2009.

17. Pesquisa Nacional por Amostra de

Domicílio, 2006. Instituto Brasileiro de Geografia e Estatística. ftp://

ftp.ibge.gov.br/Indicadores_Sociais/

Sintese_de_Indicadores_Sociais_2006/

Tabelas/ Acesso em: 16/08/2009.

18. Schoen-Ferreira TH, Silva DA, Farias

MA, Silvares EFM. Perfil e principais queixas dos clientes encaminhados ao Centro de Atendimento e Apoio

Psicológico ao Adolescente (CAAA)
UNIFESP-EPM. Psicologia em estudo. 2002; 7 (2): 73-82.

19. Linhares MBM, Parreira VLC, Maturano AC, Santa'anna SC. Caracterização dos motivos de procura de atendimento infantil em um serviço de psicopedagogia clínica. 1993; Medicina 26 (2):148-60.

20. Cabral E, Sawaia SM. Concepções e atuação profissional diante das queixas escolares: os psicólogos nos serviços públicos de saúde. Estudos de Psicologia. 2001; 6 (2): 143-155.

21. Assis SM, Avanci JQ, Pesce RP, Ximenes LF. Situação de crianças e adolescentes brasileiros em relação à saúde mental e à violência. Ciência e Saúde Coletiva. 2009; 14 (2): 319-361.

enviado em 16 de junho de 2008. modificado em 16 de novembro de 2008. aceito em 26 de agosto de 2009. 